\title{
Abstracts
}

\section{FROM MANUSCRIPT TO MOUSE-CLICK: MOVING FROM PRINT TO ELECTRONIC GUIDELINES}

L Claase, S Phillips, S Hughes, C Norquay. Therapeutic Guidelines Limited, Melbourne, Australia

10:1136/bmjqs-2013-002293.123

Background The rapid uptake of electronic devices and information sources has enormous implications for guideline developers and implementers. In 2000, our organisation anticipated this trend and developed an electronic production system.

Objectives To develop the capacity to publish and update electronic versions of a comprehensive set of clinical guidelines.

Methods An audit of technology and resourcing requirements was undertaken, new software was commissioned, and staff with IT and electronic publishing skills were recruited. Stringent quality control and editorial processes were developed to ensure the fidelity of electronic versions. A comprehensive communications and marketing plan was implemented.

Results All guidelines, covering 14 books titles and around 3000 topics, were integrated into an electronic, searchable website with links to other quality electronic resources, tools and reference materials.- Electronic subscriptions and site licenses have grown by 200\% between 2004 and2012, and currently make up $75 \%$ of users; $25 \%$ continue to use books.

Discussion The switch from paper to computer and mobile devices presents both financial and logistical challenges and requires long-term commitment. Furthermore, as the diversity of electronic integration systems multiplies, there are challenges in keeping up with technological advances. It is clear from the uptake of our electronic guidelines that health professionals and other users want access to comprehensive, up-to-date and easyto-use electronic guidelines.

Implications for Guideline Developers/Users For guidelines to be useful in practice in 2013 and beyond, they must be available electronically and be able to interact with other electronic resources used in health settings.
MEASUREMENT INSTRUMENT FOR DETERMINANTS OF INNOVATIONS (MIDI)

M Fleuren, T Paulussen, P van Dommelen, S van Buuren. TNO, Leiden, Netherlands

10:1136/bmjqs-2013-002293.124

Background In 2002, we developed a list of 60 potential relevant determinants that are critical for a successful implementation of innovations in health care, such as guidelines. This list was a result of a review of empirical studies and a Delphi study among implementation experts. Since 2002, we used the list in eight empirical studies concerning the implementation of guidelines/programs.

Objectives The development of a generic measurement instrument for the assessment of determinants of implementation of innovations.

Methods In each study, a similar method was used to measure implementation of the guideline/program as well as the determinants. The information of the eight empirical studies were combined into one data set. Missing values in the final data set were replaced by plausible values using multiple imputation. We assessed which determinants 'predict' the implementation: separately and in combination with each other. In addition, 22 implementation experts commented on the results of the metaanalyses.

Results An instrument with 29 determinants was established; 7 are related to the guideline/programme itself; 11 to the user; 11 to the organisation and socio-political context.

Discussion This instrument is to be used before or after a guideline is introduced in order to gain insight into critical determinants that need to be targeted in order to improve implementation.

Implications for Guideline Developers/Users Since 7 determinants are related to the innovation itself (e.g. clear procedures) developers should take these into account when developing a guideline. 